\title{
Excess electron transport in cryoobjects
}

\author{
D.G. Eshchenko \\ Institute for Nuclear Research, Moscow 117312, Russia \\ E-mail: eshchenko@al20.inr.troitsk.ru \\ V.G. Storchak \\ Russian Research Centre «Kurchatov Institute» \\ 46 Kurchatov Sq., Moscow 123182, Russia \\ J.H. Brewer \\ Canadian Institute for Advanced Research and Department of Physics \\ University of British Columbia, Vancouver, B.C., Canada V6T $2 A 3$ \\ S.P. Cottrell and S.F.J. Cox \\ ISIS Facility, Rutherford Appleton Laboratory, Oxfordshire OX11 OQX, UK \\ Received February 19, 2002

\begin{abstract}
Experimental results on excess electron transport in solid and liquid phases of Ne, Ar, and solid $\mathrm{N}_{2}$ - Ar mixture are presented and compared with those for He. Muon spin relaxation technique in frequently switching electric fields was used to study the phenomenon of delayed muonium formation: excess electrons liberated in the $\mu^{+}$ionization track converge upon the positive muons and form $\mathrm{Mu}\left(\mu^{+} e^{-}\right)$atoms. This process is shown to be crucially dependent upon the electron's interaction with its environment (i.e., whether it occupies the conduction band or becomes localized in a bubble of tens of angstroms in radius) and upon its mobility in these states. The characteristic lengths involved are $10^{-6}-10^{-4} \mathrm{~cm}$, the characteristic times range from nanoseconds to tens microseconds. Such a microscopic length scale sometimes enables the electron spend its entire free lifetime in a state which may not be detected by conventional macroscopic techniques. The electron transport processes are compared in: liquid and solid helium (where electron is localized in buble); liquid and solid neon (where electrons are delocalized in solid and the coexistence of localized and delocalized electrons states was found in liquid recently); liquid and solid argon (where electrons are delocalized in both phases); orientational glass systems (solid $\mathrm{N}_{2}-$ Ar mixtures), where our results suggest that electrons are localized in orientational glass. This scaling from light to heavy rare gases enables us to reveal new features of excess electron localization on microscopic scale. Analysis of the experimental data makes it possible to formulate the following tendency of the muon end-of-track structure in condensed rare gases. The muon-self track interaction changes from the isolated pair (muon plus the nearest track electron) in helium to multi-pair (muon in the vicinity of tens track electrons and positive ions) in argon.
\end{abstract}

PACS: 72.15.Rn, 67.40.Jg, 72.20.Jv, 73.20.Jc

\section{Introduction}

The investigation of electronic conduction in condensed rare gases (CRG) is of a great fundamental and practical interest. Rare gas solids (RGS) and liquids (RGL) form a group of cryosystems and cryoliquids characterized by very weak Van der Waals interatomic interactions. The forbidden gap of CRG is extremely wide (about 10-20 eV). From a fundamental point of view condensed rare gas (CRG) is a prototype of an ideal dielectric and a good object to test various theoretical approaches to the excess electrons transport in insulators. Rare gas liquids represent relatively simple disordered materials, and the study of the excess elec- 
tron transport in these systems is of considerable importance for general understanding of the electronic properties of noncrystalline solids.

On the other hand, condensed rare gases are frequently used as insulators in high-voltage devices and the processes leading to electric breakdown are certainly determined by the transport of the charge carriers in these substances. An electronic conduction in rare gas liquids is of special interest because of their employment in high energy physics experiments as working media for ionization chambers and other particle detection systems [1]. The excess electron transport in insulators is of great practical interest as it may cause electrical breakdown even in wide-gap insulating materials subjected to high electric field. These materials are used in a large number of applications ranging from power generation equipment to microelectronic devices. Nonpolar cryocrystals are used as moderators to produce ultra-low energy muon beams [2]. The yield of ultra-low energy muon could be connected with end-of-track electrons transport to the low-energetic muon. Therefore studying of the mechanisms of electron transport in solid and liquid rare gases and cryosystems is of primary importance in condensed matter physics.

Electron transport in condensed rare gases has been studied extensively for over forty years. The majority of results on the charges motion were obtained using time-of-flight (TOF) technique. In this technique the drift velocity achieved by the group of charges in an electric field is estimated by the drift length divided by the traveling time.

The time-of-flight results for low-field electron mobility in condensed $\mathrm{He}, \mathrm{Ne}, \mathrm{Ar}, \mathrm{Kr}$, and $\mathrm{Xe}$ [3] are summarized in Table 1.

Table 1

Low-field electron mobility in condensed He, Ne, Ar, Kr, and Xe [3]

\begin{tabular}{c|c|c|c}
\hline \hline \multirow{2}{*}{ Rare gas } & \multirow{2}{*}{ Triple point, $\mathrm{K}$} & \multicolumn{2}{|c}{ Electron mobility, $\mathrm{cm}^{2} / \mathrm{V} \cdot \mathrm{s}$} \\
\cline { 3 - 4 } & & in solid & in liquid \\
\hline \hline \multirow{2}{*}{$\mathrm{He}$} & - & $10^{-5^{*}}$ & $0,019^{* *}$ \\
$\mathrm{Ne}$ & 25 & 600 & 0,0016 \\
$\mathrm{Ar}$ & 84 & 1000 & 475 \\
$\mathrm{Kr}$ & 116 & 3600 & 1800 \\
$\mathrm{Xe}$ & 161 & 4000 & 1900 \\
\hline \hline
\end{tabular}

One can notice a huge difference between light and heavy CRG. In heavy CRG ( $\mathrm{Ar}, \mathrm{Kr}$, and $\mathrm{Xe}$ ) the electron mobilities were found to be of the same order of magnitude as those in conventional semiconductors $\left(\sim 10^{3} \mathrm{~cm}^{2} \mathrm{~V}^{-1} \mathrm{~s}^{-1}\right)$, implying the existence of extended delocalized electron states (band states) $[3,4]$. Low electron mobility in light RGL has been interpreted [3] as arising from electron localization in a «bubble». This bubbles are rather macroscopic objects. In liquid He the radius of the bubble is about 10-20 A [5]. Such bubbles form because of the Pauli exclusion principle: a space is opened up around the excess electron by a strong short-range repulsive exchange interaction between it and the electrons of host atoms which is opposed by weak long-range attractive interaction (caused by the polarizability of host atoms), pressure-volume interaction and surface tension. Electron bubbles are also formed in solid helium [6].

Under the increasing the atomic number the polarizability of rare gases grows up, which makes the delocalized state energetically preferable. Liquid neon $(\ell-\mathrm{Ne})$ represents a borderline case where theoretical calculations [5] failed to make definite predictions of the excess electron state. However, early time-of-flight experiments $[7,8]$ revealed only low mobility negative carriers in liquid $\mathrm{Ne}$ which were identified as stable electronic bubbles. Recent TOF experiments in high electric fields has revealed a peculiar situation when both localized and short-lived $\left(\tau \sim 10^{-9} \mathrm{~s}\right)$ delocalized electron states exist [9].

Formation of the bubble is not the only mechanism of the electron localization in cryosystems. One of the possible channels for localization of a particle is through its interaction with lattice excitations (phonons, librons, magnons, etc.). In a dissipative environment [10] the lattice excitations can be represented as a bath of harmonic oscillators; interaction with this environment causes a crossover from coherent quantum tunneling to incoherent hopping dynamics when the particle «dressed» with the lattice excitations can be effectively thought of as a polaron.

At low temperatures, the environmental excitations are frozen out. In this case, conventional understanding suggests that the only possible channel for particle localization is the introduction of crystal disorder, which thus may dramatically change the transport properties of a solid. A well-known example is the spatial localization of electron states near the Fermi-level in a disordered metallic system, which leads to a transition into a dielectric state (the Anderson transition) [11]. The concept of Anderson localization suggests that the wave function of a particle in a random potential may change qualitatively if the randomness becomes large enough. Coherent tunneling of a particle is possible only between levels with the same energy (e.g. between equivalent sites); in the case of strong 
randomness, states with the same energy may be too distant (spatially separated) for tunneling to be effective.

In metals, however, electron-electron interactions dramatically modify the density of states at the Fermi-level, leading to formation of the Coulomb pseudogap [12]. To observe the effects of disorder on electron transport without the complications of electron-electron interactions, one must therefore study electron dynamics in a disordered insulating host [13]. Orientational glasses formed by random mixtures of molecular $\left(\mathrm{N}_{2}, \mathrm{CH}_{4}, \mathrm{CD}_{4}\right)$ and atomic $(\mathrm{Ar}$, $\mathrm{Kr}$ ) species [14] offers a unique opportunity for such studies.

There is one basic drawback of TOF measurements. In such experiments the drift length between electrodes is macroscopic (typically $>10^{-2} \mathrm{~cm}$ ). This macroscopic length makes it very difficult to measure the mobilities in cryocrystals at the temperatures significantly lower the melting temperature. Under the lowering the temperature, the amplitude of the recorded current drops drastically [3]. The thermal stretch of cryocrystals results in the generation of internal strengths and defects, which can trap electrons. Moreover, the thermal stretch may result into the loss of the direct contact between the electrodes and crystal. The usage of special flexible electrodes [4,15] enabled to measure the electrons mobility down to $66 \mathrm{~K}$ in solid $\mathrm{Ar}$ (triple point $T_{3}=84 \mathrm{~K}$ ), while no experimental TOF data were obtained at lower temperatures. In liquids, where the electrode-sample contact is good, the drift velocity measurements are influenced by the presence of electron attaching impurities. If the attaching time is comparable with drift times, it is difficult to extract the drift time from the shape of damping current signal.

\subsection{Delayed muonium formation}

The techniques of muon spin rotation/relaxation/resonance $(\mu \mathrm{SR})[16]$ are of intensive use as a powerful tool providing valuable information on various chemical and solid state physics phenomena. In such experiments the beam of high (several-MeV) energy muons is injected into the sample. In many substances the muon can pick up an electron to form hydrogen-like muonium $\left(\mathrm{Mu} \equiv \mu^{+} e^{-}\right)$atom. Muon and muonium signals are well resolved in experiments due to the big difference of muon / muonium hyromagnetic ratios: $\gamma_{\mathrm{Mu}} / \gamma_{\mu^{+}} \sim-103 ; \gamma_{\mu^{+}}=13.55 \mathrm{kHz} / \mathrm{G}$. Usually there are distinguished two different ways of muonium formation - prompted and delayed. According to the first approach muonium is formed under the slowing down of energetic muon. During slowing down to an energy of a few tens of keV's, the inelastic muon scattering mainly involves the production of excitations and ionizations. At lower energies, the muon undergoes cycles of electron capture and subsequent electron loss. When the last such collision leaves atomic muonium in its neutral charge state, the muonium is said to have been formed promptly [17]. When the muon thermalizes in condensed matter as the positive ion, it leaves behind an ionization track of liberated electrons and ions. In many hosts (see for recent review [18]) some of the excess electrons generated in this track can reach the stopped muon within the time scale of the muon lifetime $\left(\tau_{\mu} \sim 2.2 \cdot 10^{-6} \mathrm{~s}\right)$ and form muonium. Essential point of this delayed muonium formation (DMF) scenario is that at «zero moment» electrons and muon are spatially separated. This circumstance enabled to create experimental procedure [19] to distinguish delayed and prompt muonium formation. DMF is sensitive to the relatively weak (up to tens $\mathrm{kV} / \mathrm{cm}$ ) external electric field which prohibits muon - electron recombination and diminishes «delayed» muonium fraction, while to affect on prompt muonium formation electric fields about atomic value are required.

\subsection{DMF and $\mu S R$ signal}

To compare experiments on muonium formation measured at different magnetic fields it is convenient to analyze general expression of muon polarization function $P(t)$ which is the experimental value of interest involved in $\mu \mathrm{SR}$ spectrum:

$$
N(t)=N_{\text {norm }} \exp \left(-t / \tau_{\mu}\right)(1+a P(t))+N_{0},
$$

where $N(t)$ is the number of muon decays registered during the time interval $[t-\delta t / 2, t+\delta t / 2]$ after muon stop ( $\delta t$ is the time per chanel for data acquisition system), $N_{\text {norm }}$ is normalization constant, $\tau_{\mu}=$ $=2.197 \cdot 10^{-6} \mathrm{~s}$ is muon lifetime, $N_{0}$ is background signal and $a$ (usually about $0.2-0.22$ ) is apparatus asymmetry. If $n(t)$ is the probability of muonium formation at the moment $t$ (where $\int_{0}^{\infty} n(t) d t$ is muonium fraction) then polarization function will be:

$$
\begin{aligned}
& P(t)=1 / 2 \int_{0}^{t} n\left(t^{\prime}\right) \cos \left(\omega_{\mu} t^{\prime}+\omega_{\mathrm{Mu}}\left(t-t^{\prime}\right)\right) d t^{\prime}+ \\
& +\left(1-\int_{0}^{t} n\left(t^{\prime}\right) d t^{\prime} \cos \omega_{\mu} t\right) .
\end{aligned}
$$

The first term in (2) is the signal from all muoniums formed up to the time $t$ including the phase shift in muon state (the constant $1 / 2$ appears 
as only triplet spin state of muonium is seen [14], the second term is the muon signal. From Eq. (2) it is clear how to extract muon fraction from experimental signal. Muon fraction is $1-\int_{0}^{\infty} n(t) d t$ or the amplitude of non relaxing signal at muon frequency. The correct procedure to extract muon fraction is to fit experimental spectrum by expression which contains non relaxing signal at muon frequency. Of course this procedure is valid if there is no other reason for muon relaxation (such as magnetic impurities, radical formation etc.), but was shown to be applicable for CRG.

Muonium fraction can be extracted from the experimental spectrum easily only in the case of «short» muonium formation (time scale of muonium formation $\tau_{\text {delay }}$ is less than muon lifetime). Then in the limit of low magnetic fields muonium fraction tends to the amplitude of the fittingat muonium frequency. In high transverse magnetic fields, where muonium signal (first term in (2)) is very small, the amplitude of relaxing signal at muon frequency equals to muonium fraction (and is twice bigger than the amplitude of muonium signal in the limit of low magnetic field).

\subsection{DMF as a tool to study microscopic electron transport}

The mechanism of DMF is critically dependent on the electron mobility and, as the characteristic muon-track electron distances are about $10^{-6}-10^{-4} \mathrm{~cm}$, represents ready to use technique of the electron mobility measurements on microscopic scale [20]. Measurements of the electric field dependences of both $\mathrm{Mu}$ and diamagnetic fractions provide information on the characteristic distance $R_{\text {char }}$ between the $\mu^{+}$and its radiolysis electrons. Due to the well known dephasing effect, the time scale of $\mathrm{Mu}$ formation $\tau$ is readily determined by measurements of the magnetic field dependence of the $\mathrm{Mu}$ precession amplitude $[21,22]$. If the muonium formation time happened to be bigger than the time resolution of $\mu$ SR spectrometer, $\tau$ can be estimated directly from the dumping rate of diamagnetic fraction [19,22]. Taking appropriate model of muon-electron recombination, from $R_{\text {char }}$ and $\tau$ one can estimate the microscopic mobility. In simple case (the viscous motion of the nearest track electron to the muon under the Coulomb attraction) this estimate is:

$$
b=\frac{R_{\text {char }}^{3} \varepsilon}{3 e \tau},
$$

where $b$ is the mutual electron-muon mobility; $\varepsilon$ is the dielectric constant of media.
The advantages of $\mu \mathrm{SR}$ technique were demonstrated in experiment with solid nitrogen [20] where, in contradiction with TOF experiments, the delocalized electrons were identified in $\alpha$ nitrogen (at $T=$ $=20 \mathrm{~K}$ ). In $\beta$ nitrogen (at $T=59 \mathrm{~K}$ ) both techniques give close estimates of the excess electron mobility; $\mu \mathrm{SR}$ experiments [23] have found a delocalized electron state in liquid $\mathrm{Ne}$, which is consistent with recent TOF measurements [9].

\section{End-of-track structure}

In a lot of substances, DMF can be artificially divided into two stages. The first stage is the slowing down of energetic muon which is accompanied with production of energetic free electrons and other excitations of the media. In this stage tack products are hot (out of thermal equilibrium with the media). The time scale of first stage is less than $10^{-10} \mathrm{~s}$. The second stage is the recombination of the track products and muonium formation itself due to the recombination of muon with one of the tracks electrons and has unlimited time-scale. From this point of view, first stage prepares «initial» conditions for the second. This include initial spatial and momentum distributions of the electrons, cations, and muon just after the end of the muon slowing down process.

To calculate initial track structure the cross sections of all elastic and inelastic track processes are to be known. For high muon energies (above about $35 \mathrm{keV}$, where the velocity of muon exceeds that of the electrons bound in the molecules of the target) the initial track structure can be calculated using the Bethe [24] theory to describe the inelastic scattering of the muon. Unfortunately the cross sections for lower energies of muon scattering are unknown. Due to the big muon to electron mass ratio the angular scattering is not essential at high muon energies and track is linear along initial muon momentum. At low muon energies muon angular scattering could play significant role. Nevertheless, experiments [18] reveal asymmetric dependences of muon (muonium) fraction vs direction of external electric field. This can be interpreted as the track products are not symmetrical around slowed down muon or the scattering of muon at low energies is not completely randomized. According to multi-pair track model $[25,26]$ the initial structure of the final part of muon track is the linear chain of cations (with muon at the end) surrounded with a cloud of electrons. In this model the influence of external electric field on muonium formation is asymmetric. At positive fields (direction of external electric field coincides with initial muon momentum) we pull track electrons from muon diminishing muonium formation. At negative fields we move «early» track 
electrons towards muon, and, in the first approximation, muonium formation should not be sensetive to «weak» electric field.

As it will be shown later, this simplified model is a good start to understand muonium formation in $\mathrm{CRG}$ and other condensed gases. There are three characteristic distances in this model: the distance between cations in the chain $R_{+}$, the distance between the last cation and muon $R_{\mu}$, and the square average distance from the scattered electron to the parent cation $R_{e}$. The fact that in CRG at zero electric field muonium fraction is big [18] or the probability of muon-electron recombination is high implies $R_{e}>R_{+}$and $R_{e}>$ $>R_{\mu}$. It means that for all CRG under consideration muon is inside its own track. Otherwise electron will rather recombine with one of positive ions. The number of electrons involved in muonium formation at zero and positive fields will be $N_{e} \sim R_{e} / R_{+}$. The characteristic fields to suppress muonium formation will be order of $E_{\mathrm{char}_{+}} \sim e / \varepsilon R_{e} R_{+}$.

At negative fields muonium formation is sensitive to the flux of «early» track electrons an one should expect the track motion to be different at fields higher $E_{\text {char }} \sim e / \varepsilon R_{+}^{2}$ where we can neglect interactions between anions.

Details of the track motion in different CRG will be discussed in corresponding sections.

Table 2

Muonium fraction in condensed $\mathrm{He}$ [27], Ne, and Ar

\begin{tabular}{c|c|c}
\hline \hline \multirow{2}{*}{ Rare gas } & \multicolumn{2}{|c}{ Muonium fraction } \\
\cline { 2 - 3 } & in solid & in liquid \\
\hline \hline${ }^{4} \mathrm{He}$ & 0 & $0,92^{*}$ \\
$\mathrm{Ne}$ & 0,80 & $0,80^{* *}$ \\
$\mathrm{Ar}$ & 0,90 & 0,90 \\
\hline \hline
\end{tabular}

C o m m e n t: ${ }^{*} T>0.8 \mathrm{~K} ;{ }^{* *} T=25 \mathrm{~K}$

\section{Experimental details}

Experiments in condensed $\mathrm{He}$ are described elsewhere [19,22,27] (see Table 2). Experiments in condensed $\mathrm{Ne}, \mathrm{Ar}$, and $\mathrm{N}_{2}-\mathrm{Ar}$ were performed at M13 spin-polarized surface muon beam line at TRIUMF and EMU pulsed spin-polarized muon beam line at ISIS (Rutherford Appleton Laboratory). In both laboratories we used similar sample cells. Front and back sides of brass sample cell $(22 \mathrm{~mm}$ inner diameter for TRIUMF setup and $35 \mathrm{~mm}$ inner diameter for ISIS and $5 \mathrm{~mm}$ thick) are glued with $0.1 \mathrm{~mm}$ titanium windows. This thickness is small enough to estimate the stops of surface muons (momentum $\approx 28 \mathrm{MeV} / \mathrm{c}$ ) in incoming foil within few percent. Muon beam was collimated down to $9 \mathrm{~mm}$ at TRIUMF experiments. At ISIS the spot size of muon beam is about $20 \mathrm{~mm}$; therefore essentially all the muons were stopped in samples in both laboratories.

Windows are glued trough $0.5 \mathrm{~mm}$ kapton layings (with inner diameter $0.5 \mathrm{~mm}$ less than the diameter of the cell) by epoxy and supported by brass flanges. Such a construction enables to apply high voltage (up to tens $\mathrm{kV}$ ) on any window and was tested at pressure $10 \mathrm{~atm}$ at room temperature. To produce temperature gradient required for crystal growth at the top and at the bottom of sample space there are special copper plates $(0.5 \mathrm{~mm}$ thick $)$. Bottom plate is connected to the cold finger of the cryostat. Top plate is placed at capillary insert which is connected to gradient heater. Both plates are attached to the cell body with little gap (about half millimeter). This enables good thermal contact to the bulk of the solid CRG sample under lowering the temperature.

We used standard high purity (impurity content $\leq 10^{-5}$ ) gases. Samples were condensed from big ballast volumes by cooling the cell. Crystallization of the samples was made by steady lowering the base temperature with open ballast volume (constant pressure regime). Typical crystal growth times were about one hour, temperature gradients were about $0.5 \mathrm{~K}$ per sample length.

Muon experiments in solid condensed gases are connected with some experimental difficulties. First - in spite of high electrical strength of balk solid condesed gases, under the lowering the temperature below melting point thermal cracks of the sample and small equilibrium pressure of the gas vapor result in the appearance of «easy ways» for electrical breakdown of the sample. To increase the maximum electrical strength of our solid condesed gases samples we added some pressure (up to $1 \mathrm{~atm}$ ) of He gas into the sell just after the cooling below melting point. Unfortunately this procedure is effective only at temperatures below superfluid $\lambda$ transition $T_{\lambda}=2.17 \mathrm{~K}$, where superfluid film can penetrate into all the sample cracks. At higher temperatures, the procedure of $\mathrm{He}$ gas filling helps to increase the breakdown voltage about two times. In solid neon we succeeds to increase the maximum electric field up to $12 \mathrm{kV} / \mathrm{cm}$ which is still less than the maximum electric field in liquid neon $\left(E_{\max } \sim 33 \mathrm{kV} / \mathrm{cm}\right)$.

Our first attempts to increase the range of electric field were accompanied with the huge scattering of the experimental data. Now we understand that this problem is connected with sample-electrode interface. The incoming muon beam eventually produces the ra- 
diation damage of the sample, in particular, it generates free carriers (electrons and ions). If there is a barrier for the electrons to pass trough the electrode-sample gap (which is probably formed under the cooling of the sample) under the influence of external electric field it will result in the accumulation of the space charge near the sample surface. This charge screens the external electric field inside the sample (if not cancel it completely) which may diminish (if not cancel) the effect under study.

The time scale of this screening will depend upon the free carrier generation rate in the bulk of the sample and on the electrical properties of the sample-electrode connection. For typical muon flux on accelerators, the time scale range from tens of minutes to seconds. As the typical time for collecting the necessary statistics in one spectrum is about one hour the screening effect can mask the effect of electric field. To avoid this difficulty we have developed a special technique of $\mu \mathrm{SR}$ measurements in alternating electric fields [28]. If the frequency of the alternating field is higher than the reverse time of the screening charge accumulation near the sample surface, the internal electric field in the sample tends to applied external electric field. For example in solid Ne for the incoming muon beam intensity of about $3 \cdot 10^{4} \mathrm{~s}^{-1}$ the switching period should not exceed 5 seconds.

\section{Results and discussion}

\subsection{Liquid and solid helium}

To represent complete picture of end-of-track processes in CRG we start from reproduction of $\mu \mathrm{SR}$ results in condensed helium $[19,22,29]$ where the features of DMF are seen in most clean form.

The main reason of this is the unique property of liquid helium - the phenomenon of superfluidity. The mobility of the charge carriers is relatively low $\left(b_{+}=0.05 \mathrm{~cm}^{2} \mathrm{~V}^{-1} \mathrm{~s}^{-1}\right.$ for cations and for anions $b_{-}=$ $=0.03 \mathrm{~cm}^{2} \mathrm{~V}^{-1} \mathrm{~s}^{-1}$ ) in normal helium [30] at $4.2 \mathrm{~K}$. The spectrum of elementary excitations in the superfluid helium has a characteristic threshold, which causes an exponential increase of the mobility [31] as the temperature is reduced below the lambda point $T_{\lambda}=2.17 \mathrm{~K}\left(b_{+}=1700 \mathrm{~cm}^{2} \mathrm{~V}^{-1} \mathrm{~s}^{-1}\right.$ for cations and $b_{-}=155 \mathrm{~cm}^{2} \mathrm{~V}^{-1} \mathrm{~s}^{-1}$ for anions at $0.6 \mathrm{~K}$ ). This circumstance gives unique opportunity to examine various models of track motion by scaling the mobility over five orders of magnitude. Moreover, by adding a small amount of ${ }^{3} \mathrm{He}$ one can regularly diminish the mobilities at low temperatures $T<0.8 \mathrm{~K}$ (where the mobilities are determined by the scattering at ${ }^{3} \mathrm{He}$ impurities) keeping the other parameters (temperature etc.) unchanged. Unfortunately, the behavior of cati- ons and anions in helium is outstanding in comparison with other CRG (especially heavy CRG). First, the charges are extremely heavy: electrons form «bubbles» with hydrodynamic mass about 200 helium atoms, positive charges form «snow balls» about 40-60 helium atoms [32]. Second, in all the temperature interval in liquid the mobility of anions is bigger then the mobility of cations, while in solid heavy CRG the mobility of anions is negligible in comparison with the mobility of light delocalized electrons.

This fact will result in the breakdown of the linear structure of track anions chain. If we suppose $R_{e}>$ $>R_{+}$and $R_{e}>R_{\mu}$, then just after the slowing-down process (when electrons have formed bubbles, positive helium atoms and, probably, muon have formed snowballs) the main interaction between the charges will be the strong plus-plus repulsion. For example, muon will feel high repulsion force from the nearest positive ion or even (if $R_{+}<R_{\mu}$ ) group of $R_{e} / R_{+}$ ions in the direction preliminary parallel to the primary muon beam. Muon-positive ions repulsion can explain the origin of «initial» muon snowball momentum $M V_{L}$ (where $M \sim 40 m_{\mathrm{He}}$ is the mass of snowball, $V_{L} \sim 6 \cdot 10^{3} \mathrm{~cm} / \mathrm{s}$ is Landay velocity) required to explain the decrease of muonium fraction at temperatures $T<0.7 \mathrm{~K}$ in pure ${ }^{4} \mathrm{He}$ [19].

Repulsion of light and mobile anions will lead for a short time interval all the distances between all the charges to become the same order of magnitude about $R_{e}$. Then the process of muonium formation continue in long (in comparison with plus-plus repulsion) recombination of $\mu^{+}$and $e^{-}$in simple Coloumb potential.

Note that single muon - electron pair will also arise if hot excited muonium decays far away from other track (muon-electron pair is far from track from the very beginning of the track recombination). Minor point of this scenario is the difficulty to explain asymmetry of muon-electron initial position which is readily obtained in multi-pairs model.

Combining all mentioned above one can conclude, that the longest part of muon-electron interaction in liquid helium can be written in the simplest muon-the nearest electron Coloumb attraction no matter what is the origin of this pair. This approach allowed to draw qualitative (and some time a quantitative) description of muonium formation in helium [19]. Let $r_{0}$ is the typical distance between muon and electron and $b=$ $=b_{+}+b_{-}$is the sum of charges mobilities. The mobility of muon is supposed the same as for «heavy» positive charges mobility [31]. In the normal helium the mobility of charges is low and the equation of mutual muon-electron motion seems to be viscous one. In this case the time of muonium formation is $\tau=\varepsilon r_{0}{ }^{3} / 3 b e$. The forming muoniums reduce the free muon fraction. 
In high transverse magnetic fields (where only muon precession signal is seen) this muonium formation causes apparent muon relaxation $\lambda \propto \tau^{-1}$ and polarization function is directly connected with the number of free muons. From the relaxation rate in normal helium $\lambda=0.06 \mu \mathrm{s}^{-1}$ and taken $b \simeq 0.08 \mathrm{~cm}^{2} \mathrm{~V}^{-1} \mathrm{~s}^{-1}$ one can estimate the typical value for muon-electron distribution $r_{0} \simeq(3 b e / \lambda)^{1 / 3}=7 \cdot 10^{-5} \mathrm{~cm}$ in liquid helium [19]. Note that Onsager length $R_{c}=\left(e^{2} / k_{b} T \varepsilon\right) \sim$ $\sim 10^{-4} \mathrm{~cm}$ at helium temperatures is much longer than $r_{0}$ so the diffusion in Mu formation is not important. As for the amplitude of muonium precession $a_{\mathrm{Mu}}(H)$, in normal He only small part of pairs manage to recombine during the time of the precession signal formation $\tau(H) \sim 1 /\left(\gamma_{\mathrm{Mu}} H\right) \sim 10^{-7}$ s for $H=0.4 \mathrm{G}$, and $\mathrm{Mu}$ precession amplitude is small. To obtain more «visible» muonium one should diminish the time of mutual muon-electron motion and use low magnetic fields. After entering the superfluid phase, $a_{\mathrm{Mu}}(0.4)$ begins to increase with lowering the temperature (accompanied with exponential growth of mobility). When $b$ becomes $10^{3}$ times that in normal helium, the most distant pairs manage to converge during the time $\tau(0.4)$, and the amplitude of muonium precession achieves its maximum value which corresponds to the muonium fraction $92 \%$. This takes place at $T=$ $=0.7-0.85 \mathrm{~K}$ in pure ${ }^{4} \mathrm{He}$. Under further lowering of the temperature in pure ${ }^{4} \mathrm{He} a_{\mathrm{Mu}}$ drops and tends to the constant about 0.8 of maximum value. This behavior can be explained taken into account initial momentum of muon snowball [19] and ballistic motion (due to extremely low drag force) of this snowball around nearest electron at temperatures $T<0.7 \mathrm{~K}$. If under the slowing down process $\mu^{+}$forms snowball at velocity higher than $M / m_{\mu} V_{L} \sim 2.4 \cdot 10^{6} \mathrm{~cm} / \mathrm{s}$ (where $M / m_{\mu} \sim 400$ is snowball to muon mass ratio) or the snowball receives momentum due to the repulsion from track cations on the first stage of track motion (see speculation in previous paragraph), then all the muons with positive energy $M V_{L}^{2} / 2-e^{2} / r_{i} \varepsilon>0$ (where $r_{i}$ is the «initial» muon-electron distance) will escape muonium formation. Appropriate estimate gives «critical» value $r_{c}$ (all the muons with initial conditions $r_{i}<r_{c}$ will definitely recombine with electron) is $r_{c}=2 e^{2} / \varepsilon M V_{L}^{2} \sim 6 \cdot 10^{-5} \mathrm{~cm}$, which is comparable with typical muon-electron distance estimated from the rate of muonium formation in normal helium $r_{0} \sim 7 \cdot 10^{-5} \mathrm{~cm}$. The real motion of charges in superfluid helium is far from classical ballistic regime. If under the influence of electric field charge exceeds critical Landay velocity it will (within timescale $10^{-13} \mathrm{~s}$ ) emit roton(s), diminish its speed [33] and follow new trajectory with reduced energy. That's why significant part of muon snowballs will form muonium even initial ener-

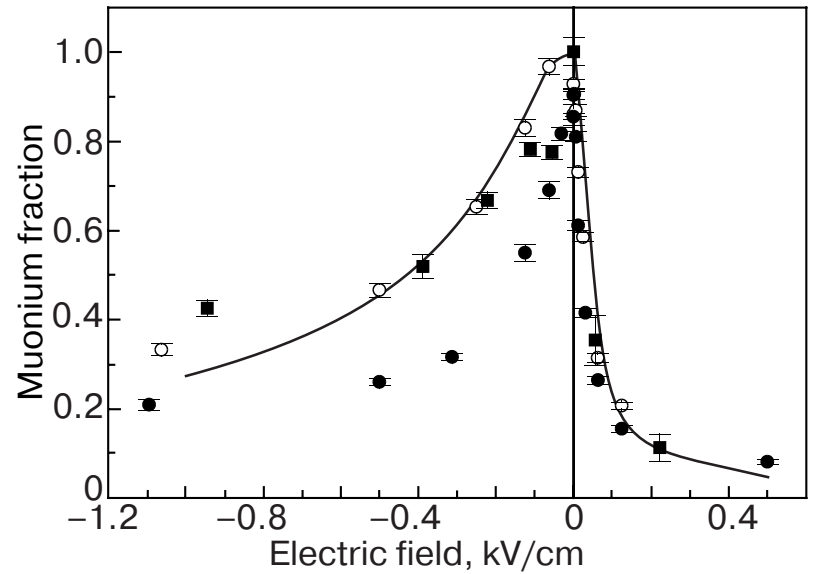

Fig. 1. Electric field dependences of muonium fraction in liquid helium: ${ }^{4} \mathrm{He}+0.2 \%{ }^{3} \mathrm{He}$ at $T=0.5 \mathrm{~K}$ (open circles); pure ${ }^{4} \mathrm{He}$ at $T=1.7 \mathrm{~K}$ (solid squares); pure ${ }^{4} \mathrm{He}$ at $T=0.7 \mathrm{~K}$ (solid circles). The direction of electric field coincides with initial momentum of muon beam. Solid line represents the best fit of the viscous model to the data at $T=0.5 \mathrm{~K}$ in mixture (see text).

gy is positive. In mixture ${ }^{4} \mathrm{He}+0.2 \%{ }^{3} \mathrm{He}$, where due to the scattering at ${ }^{3} \mathrm{He}$ impurities the low temperature anion mobility can not exceed $20 \mathrm{~cm}^{2} \mathrm{~V}^{-1} \mathrm{~s}^{-1}$, the motion of muon snowball is the viscous one, and there is no peculiarity in temperature dependence of muonium fraction [19].

The most powerful tool to study muonium formation are experiments in electric fields. External electric field prevents recombination of track charges, diminishes muonium fraction and increases muon fraction.

The electric field dependence of double amplitude of muonium precession divided to apparatus asymmetry in diluted mixture $-{ }^{4} \mathrm{He}+0.2 \%{ }^{3} \mathrm{He}(T=0.5 \mathrm{~K})$ measured in low magnetic field $H=0.4 \mathrm{G}$ [19] is represented on Fig. 1 by open circles. Under such conditions this normalized amplitude tends to muonium fraction (see section DMF and $\mu$ SR signal), at least $90 \%$ of muons recombine, forming $\mathrm{Mu}$ in less than $10^{-7} \mathrm{~s}$, and the double amplitude of muonium precession $a_{\mathrm{Mu}}$ in zero electric field is close to its utmost value $a_{\mathrm{Mu}}^{\max }=$ $=0.2[22]-$ the apparatus asymmetry.

At high temperatures close to the $\lambda$ point, the time of muonium formation in liquid helium is about $5 \cdot 10^{-6} \mathrm{~s}$ and the amplitude of muonium signal is low even at fields $H=0.4 \mathrm{G}$. To represent muonium fraction at $T=1.7 \mathrm{~K}$, the amplitude of relaxing signal at muon frequency divided to apparatus asymmetry is plotted on Fig. 1 by solid squares. One can notice that in spite of huge difference of the mobilities of the charges at these temperatures $\left(b=20 \mathrm{~cm}^{2} \mathrm{~V}^{-1} \mathrm{~s}^{-1}\right.$ at $0.5 \mathrm{~K}$ and $b=0.2 \mathrm{~cm}^{2} \mathrm{~V}^{-1} \mathrm{~s}^{-1}$ at $\left.1.7 \mathrm{~K}\right)$ the electric field dependences of muonium fractions are very si- 
milar. This behavior suggests similar distribution of the tracks products (or, by other words - the same initial electron-muon distribution function) in all the temperature interval $0.5-4.2 \mathrm{~K}$ in ${ }^{4} \mathrm{He}+0.2 \%{ }^{3} \mathrm{He}$ mixture. The case of mixture is very important as the main mechanism of muon cluster scattering at low temperature is guided by impurity $\left({ }^{3} \mathrm{He}\right)$ atoms with momentum $p_{i} \sim\left(m_{3} k T\right)^{1 / 2}$. Therefore the mean path between collisions of a cluster is $L=\Delta p b / e \sim 3 \cdot 10^{-7} \mathrm{~cm}$ (where $\Delta p \sim p_{i}$ is the change of cluster momentum in a single collision) is small in comparison with typical length in the problem. Then the motion of muon cluster will be of «viscous» type - the direction of the cluster motion will coincide with the direction of an electric field which acts on it. For any given configuration of electric field (internal track field plus external field) it will result to the separation in the space of initial electron-muon pairs into two regions. In the first region, where the lines of electric field strength end at muon, muon will form muonium. In the second, where the lines of electric field strength go to infinity, muon will escape muonium formation. In the case of single electron-muon interaction, muon will recombine if

$$
r<(e / E \varepsilon)^{1 / 2} \cos (\vartheta / 2),
$$

where $r$ and $\vartheta$ are spherical coordinates (vector $\vartheta=0$ coincides with the direction of the external electric field), and will be withdrawn by external field otherwise. Solid line on Fig. 1 represents the best fit of the calculated (using criterion (4)) normalized muonium amplitude to the measured values at $T=$ $=0.5 \mathrm{~K}$ in ${ }^{4} \mathrm{He}+0.2 \%{ }^{3} \mathrm{He}$ mixture. Initial mutual muon-electron distribution function was searched in form of three dimensional Gaussian [19]:

$$
\begin{aligned}
& p(\mathbf{r})=(2 \pi)^{-3 / 2} \sigma_{x}^{-1} \sigma_{y z}^{-2} \times \\
& \times \exp \left[\frac{-(x-a)^{2}}{2 \sigma_{x}^{2}}\right] \exp \left[\frac{-\left(y^{2}+z^{2}\right)}{2 \sigma_{y z}^{2}}\right],
\end{aligned}
$$

where $x$ is parallel to the external electric field, point $(\mathrm{a}, 0,0)$ is the center of muon-electron distribution, $\sigma_{x}$ and $\sigma_{y z}$ are appropriate dispersions of initial $\mathrm{mu}^{-}$ on-electron position. The best fit gives $a=4 \cdot 10^{-5} \mathrm{~cm}$ (the maximum of muons density is shifted forward in the direction of $p_{\mu}$ ) with respect to the electrons), $\sigma_{x}=4 \cdot 10^{-5} \mathrm{~cm}, \sigma_{y z}=2 \cdot 10^{-5} \mathrm{~cm}$.

In the pure helium at low temperatures, electric field has stronger effect than in mixture. The electric field dependence of normalized muonium amplitude in pure ${ }^{4} \mathrm{He}$ is represented on Fig. 1 by solid circles. This difference is apparently due to the high mobility $\left(b_{+} \simeq 200 \mathrm{~cm}^{2} \mathrm{~V}^{-1} \mathrm{~s}^{-1}\right)$ of the particles in pure helium. Under this conditions viscous approach of the muon cluster motion is not valid (mean free path becomes comparable with the dimension of the problem) and one should take into account initial momentum of the cluster. In the case when $\mu^{+}$starts with velocity pointing from $e^{-}$, muon can go very far away from electron. For initial distance $r_{0}>2 e^{2} / m v_{L}^{2} \simeq 5 \cdot 10^{-5} \mathrm{~cm}$ (where the kinetic energy of the cluster is greater than the potential electrostatic energy) the maximum charges separation will amount to $r_{\max } \sim r_{(0)}+v_{L} \mathrm{mb} / e \simeq$ $\simeq 2 \cdot 10^{-4} \mathrm{~cm}$, and the effect of the field will be seen already at the magnitude $E>e / r_{\text {max }}^{2} \simeq 5 \mathrm{~V} / \mathrm{cm}$.

The mobilities of the charges are extremely low $\left(b<10^{-5} \mathrm{~cm}^{2} \mathrm{~V}^{-1} \mathrm{~s}^{-1}\right)$ in solid helium [34]. This is the reason of complete absence of muonium signal in solid ${ }^{4} \mathrm{He}$ [29]. Moreover, the muon signal shows no damping $-\lambda_{\text {muon }}<0.004 \cdot 10^{-6} \mathrm{~s}^{-1}$, which means the high limit of the muonium formation time about $\tau \sim 10^{-4} \mathrm{~s}^{-1}$.

As a conclusion of this section we will summarize the main features of muonium formation in condensed helium: high relative mobility of positive ions leads to the break down of the linear structure of muon track and results in «single pair» regime of muon-nearest electron attraction; in the wide range of the charges mobilities (until viscous limit of the track motion is valid) the mutual «initial» distribution of the track products is independent on temperature; low mobilities of ions in solid helium prohibit muonium formation.

\subsection{Liquid and solid neon}

Muon spin rotation experiments in solid Ne [18,35] revealed near maximum value of muonium precession signal, which is natural taking into account high electron mobility in solid Ne (see Table 1). Electric field experiments in solid $\mathrm{Ne}$ shows a great change in muonium/diamagnetic signals, though the characteristic fields are almost ten times bigger those for liquid He. Our recent experimental data - diamagnetic signal vs electric field dependence in solid Ne near the melting point $(T=22 \mathrm{~K})$ is plotted on Fig. 2 by solid circles. One can notice significant asymmetry in the influence of electric fields (in this article, the positive sign of electric field coincides with initial muon beam momentum), which means that the electron density is shifted to the direction opposite to the initial muon momentum. High characteristic electric fields can be explained by multi-pairs structure of muon track in solid neon (see section «End-of-track structure»). In this case the intrinsic electric field on electron will be $E_{\text {char }} \sim e / \varepsilon R_{+} R_{e}$, where $R_{+}$is the distance between cations in the chain and $R_{e}$ is the square average distance from the scattered electron to the parent cation. 


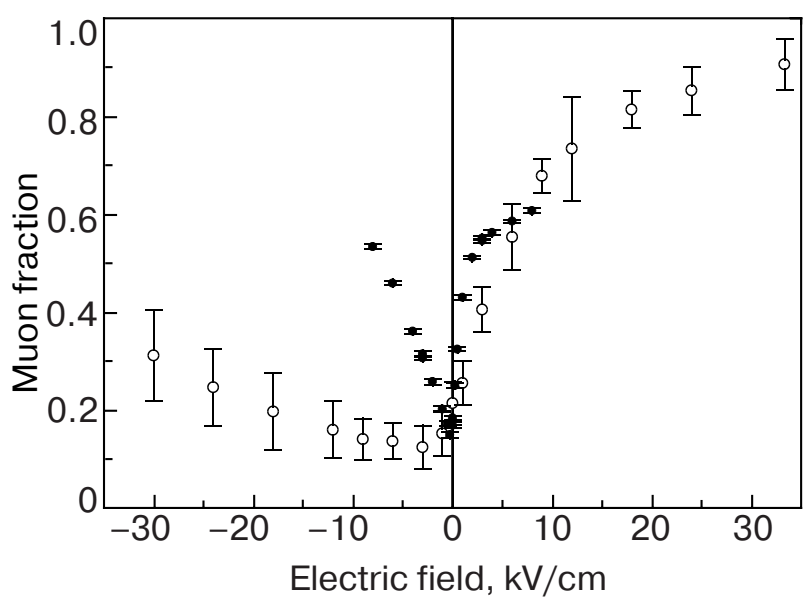

Fig. 2. Electric field dependences of muon fraction in liquid $\mathrm{Ne}$ at $T=25 \mathrm{~K}$ (open circles) and solid $\mathrm{Ne}$ at $T=22 \mathrm{~K}$ (solid circles). The direction of electric field coincides with initial momentum of muon beam.

This field is $R_{e} / R_{+}$times bigger than the single pair Coulomb interaction.

Experimental $\mu \mathrm{SR}$ signal is very sophisticated in liquid neon. It contains the signal at muonium frequency and two component (relaxing and not relaxing) diamagnetic signal. The essential feature of muonium signal is that it is in contradiction with the envelope of diamagnetic signal. The amplitude of muonium precession is much higher than expected from Eq. (2). It looks like corresponding to short process in comparison with damping rate of diamagnetic signal or even in comparison with apparatus time resolution.

It could be two possibilities for this behavior - prompt muonium or «fast» DMF. In the experiment with solid neon, due to the limited electric strength of the samples we achieved only partial recovering of diamagnetic signal (see Fig. 2). That's why from solid phase data it is unclear-is all the muonium signal delayed or some part of this signal comes from prompt muonium? It is very unlikely that cross sections for charge exchange processes are different in solid and liquid $\mathrm{Ne}$ and prompt fraction (if any) should be the same in both phases. The fact that all the signals in liquid depend on external electric field and complete suppression of muonium signal at high positive fields implies the track origin of muonium signal in liquid Ne. It was proposed [23] the coexistence of localized in bubbles and delocalized in conduction band electron states in liquid neon. In high magnetic fields $H>1 / \tau_{\text {loc }} \gamma_{\mathrm{Mu}}$ (where $\tau_{\text {loc }}$ is the characteristic time of muon-electron recombination with electron in bubble state), fast delocalized electrons give the main contribution into muonium precession amplitude. Muonium formation due to the arrival of slow localized electrons is seen in the relaxation of diamagnetic signal. The coexistence of localized and delocalized electrons in liquid neon was also observed by time-of-flight measurements [9].

The electric field dependences of normalized to the apparatus asymmetry amplitude of twice muonium $2 A_{\mathrm{Mu}}$ (circles) and relaxing diamagnetic $A_{\text {rel }}$ (squares) signal in liquid $\mathrm{Ne}$ at $T=25 \mathrm{~K}$ are plotted on Fig. 3. The sum of these two signals is muonium fraction vs electric field. The ratio $A_{\mathrm{Mu}} / \mathrm{A}_{\text {rel }}$ is the part of delocalized electrons vs electric field. It can be shown that this ratio is the increasing function of external electric field. Unfortunately we can not plot the probability of delocalization from electric field which acts on electron. This «true» electric field is external electric field plus the intrinsic track electric field, the second term we can estimate only.

For comparison with solid Ne the normalized amplitude of non relaxing diamagnetic signal from external electric in liquid neon is plotted on Fig. 2 by open circles. This value reflects the part of muons which escapes recombination. One can notice huge difference between liquid and solid Ne. In liquid in negative fields free muon fraction changes a little (at fields up to $-10 \mathrm{kV} / \mathrm{cm}$ it is practically constant), in positive fields the slope of the curve in solid is bigger those in liquid.

The possible explanation of peculiarities mentioned above could be the following. Weak dependence in liquid implies that the characteristic muon-electron distance is shorter in liquid. In both substances the excess track electrons are initially delocalized and moving rapidly away from the ionization centers where they were created. Then electrons lose there energy in collisions with the media atoms. In solid the electrons remain delocalized up to the recombination with muon

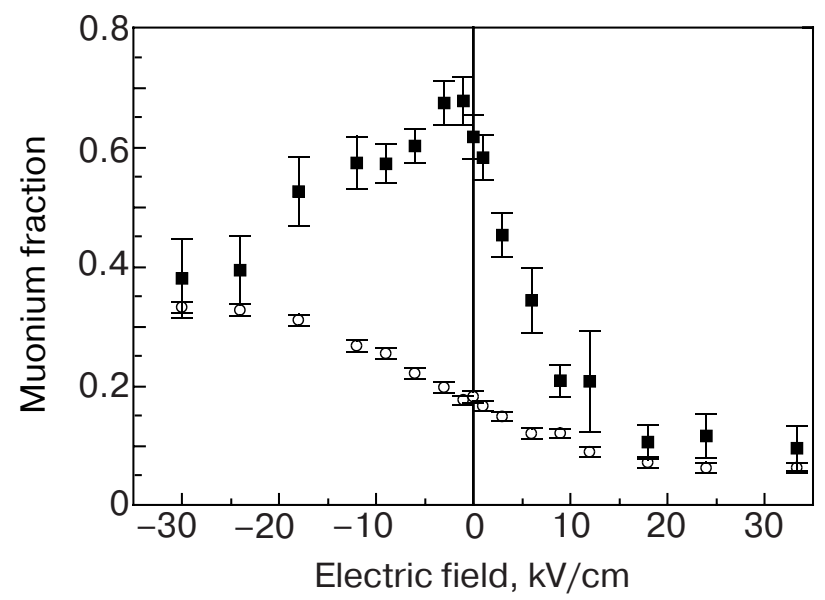

Fig. 3. Electric field dependences of muonium fractions in liquid $\mathrm{Ne}$ at $25 \mathrm{~K}$ formed by: delocalized electrons (open circles); localized electrons (solid squares). The direction of electric field coincides with initial momentum of muon beam. 
(or other positive center). In liquid under the lowering of the electron velocity the probability for the electron to be localized in «bubble» is increased [9]. It implies that the mean square distance between the delocalized electron and his parent ion is shorter in liquid or the intrinsic track electric fields are bigger in liquid neon and dependence of free muon fraction from external electric field is more flat.

At negative fields we pull «early» track electrons to the muon. The free diamagnetic asymmetry seems to be the result of the two tendencies. The higher is the field - the less is the probability for the muon to recombine with any separate electron, but the higher is the probability to find additional track electron. If the second tendency dominates, the probability of muonium formation tends to one and, in this sense, is independent on electric field.

Unless specially prepared, the solid CRG sample (excluding solid $\mathrm{He}$, which anneals easily) has a polycrystalline structure with a characteristic grain size [3] of about $10^{-5}-10^{-4} \mathrm{~cm}$. If electrons are scattered or captured by polycrystalline defects, only electrons within some critical distance (about crystalline size) will achieve muon. The amount of these electrons will be $N_{\text {neg }}=R_{\text {cr }} / R_{+}$, where $R_{\text {cr }}$ is the crystalline size, $R_{+}$is the distance between ionizations at the final stage of muon track. One should expect the negative part of $E$-field dependences to be sensitive to the quality of the crystal. In liquid crystalline like defects are absent, the amount of electrons involved in muonium formation is bigger and probability of muonium formation with «early» electrons higher.

As a conclusion of this section end-of-track processes in solid and liquid neon are very different. In solid Ne, muonium is formed as a result of muon recombination with delocalized electrons. In liquid muon can recombine with delocalized or localized electrons. Localization of initially free track electrons in liquid results in high (in compression with solid $\mathrm{Ne}$ ) internal electric fields.

\subsection{Liquid and solid argon}

Electrons are delocalized in both solid and liquid argon (see Table 1), and one should expect endof-track processes in condensed argon to be similar to solid Ne. Electric field dependences of free muon fractions in liquid at $T=84 \mathrm{~K}$ (open circles) and solid argon at $T=78 \mathrm{~K}$ (solid circles) are represented on Fig. 4. Data were obtained via $\mu \mathrm{SR}$ measurements in switching electric fields. The switching time is $10 \mathrm{~s}$. One can notice a practical coincidence of the experimental points in liquid and solid argon at positive fields and significant difference at high negative fields. At negative fields the points in liquid are lower than points in solid. Moreover, in spite of using the technique of electric field measurements in switching fields, the points at negative fields in solid were «sample dependent». Switching helps to remove random scattering of the data but keeps different slopes of the negative part of the curve measured at «different» conditions. The data represented on figure were obtained just after the crystallization of the sample. After few hours of annealing the data change to lower values closer to the points in liquid.

The peculiarities mentioned above can be explained in the framework of multi-pairs structure of muon track. The explanation is analogous to this in condensed neon with simplification that electrons are delocalized in both phases of Ar. Then the characteristic track distances seem to be close in solid and liquid argon.

If we suppose both the characteristic distance between the positive ions $R_{+}$and the distance from the last track ionization to the stopped muon $R_{\mu}$ to be less than the square average distance from the scattered electron to the parent ion $R_{e}$, then at positive fields, when we pull track electrons from the muon, the amount of particles involved in muonium formation will be $R_{e} / R_{+}$. If $R_{e}$ is bigger than crystalline size of solid sample, muonium formation will be similar in solid and liquid phases.

At negative fields we push «early» track electrons towards muon and muonium formation is sensitive to the quality of the sample. In solid the scattering or even trapping of track electrons at crystalline imperfections diminish the flux of early electrons and the probability of muonium formation is lower then in liquid. Annealing of crystalline defects will result to the increase of electron flux to the muon, and the probability of muonium formation at negative fields tends

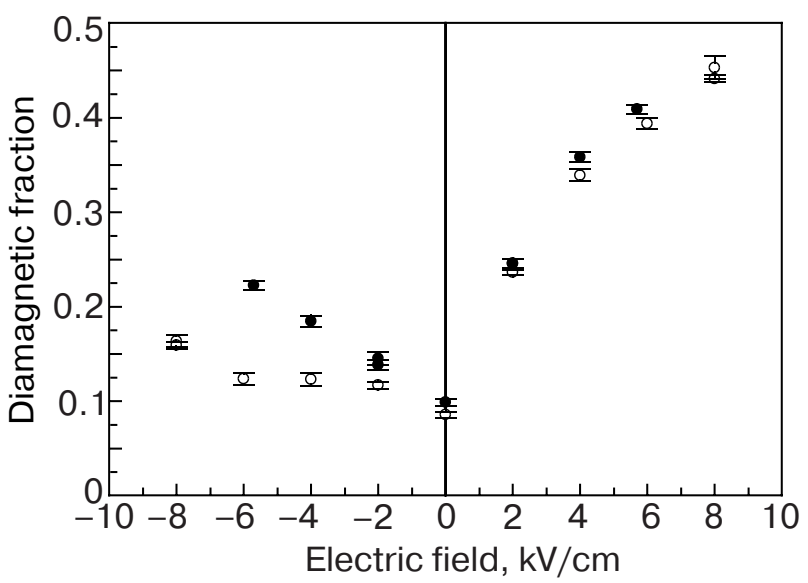

Fig. 4. Electric field dependences of muon fraction in liquid $\mathrm{Ar}$ at $T=84 \mathrm{~K}$ (open circles) and solid $\mathrm{Ar}$ at $T=78 \mathrm{~K}$ (solid circles). The direction of electric field coincides with initial momentum of muon beam. 
to the value in liquid where crystalline defects are absent.

From argon data we can estimate amount of electrons involved in muonium formation in condensed Ne. In condensed Ar muon fraction at zero field is less those in solid Ne. Appropriate values measured in the same experimental chamber at TRIUMF are $A_{\mu_{\mathrm{Ar}}}=$ $=0.076(3)$ and $A_{\mu_{\mathrm{Ne}}}=0.177(6)$. At the final stage of muon track, as we have $\mu^{+}$one additional positive particle, the probability for the muon to escape the recombination will be $A_{\mu}=1 /(N+1)$, where $N$ is the number of electrons (or positive ions) involved in muonium formation in zero electric field. It gives rough estimate for the number of the electrons: $N_{e_{\mathrm{Ne}}} \sim 1 /\left(A_{\mu_{\mathrm{Ne}}}-A_{\mu_{\mathrm{Ar}}}\right) \sim 10$. Unfortunately we can not do analogous estimate for condensed $\mathrm{Ar}$ as some unknown part of muon signal comes from thermal shields, chamber windows etc. Low value of muon fraction at zero field in Ar suggests that amount of electrons involved in muonium formation $N_{e_{\mathrm{Ar}}}$ is bigger than $N_{e_{\mathrm{Ne}}}$. As an alternative estimate of $N_{e}$ is $N_{e} \sim$ $\sim R_{e} / R_{+}$, this ratio is bigger for condensed Ar. On the other hand characteristic electric field to suppress muonium formation at positive fields is $E_{\text {char }} \propto$ $\propto e / \varepsilon R_{+} R_{e}$. This fields are close for Ar and solid Ne. Combining all mentioned above and taking $E_{\text {char }} \sim$ $\sim 4-10 \mathrm{kV} / \mathrm{cm}$ we can estimate: $R_{+_{\mathrm{Ne}}} \sim 1.5-2 \cdot 10^{-6} \mathrm{~cm}$, $R_{e_{\mathrm{Ne}}} \sim 1.5-2 \cdot 10^{-5} \mathrm{~cm}, R_{e_{\mathrm{Ar}}}>R_{e_{\mathrm{Ne}}}$, and $R_{+_{\mathrm{Ar}}}<R_{+_{\mathrm{Ne}}}$.

Due to excess electron delocalization in both phases end-of-track processes in liquid and solid (near triple point) Ar are very similar. Low value of muon fraction in zero electric field suggests multi-pair structure of muon track. The amount of particles involved in muonium formation is about tens.

\subsection{Electron localization in a disordered insulating host}

Most of our understanding of electron transport in solids is modelled on nearly perfect crystalline materials, but even in this limit disorder plays a crucial role [36]. The most familiar phenomenon governing electron transport in disordered metals is «Anderson localization» [11]: introduction of sufficiently strong disorder into a metallic system causes spatial localization of electron states near the Fermi level and thus drives a transition to an insulating state. To observe the effects of disorder on electron transport without the complications of electron-electron interactions, we studied electron dynamics in a disordered insulating host [13].

Orientational glasses formed by random mixtures of molecular $\left(\mathrm{N}_{2}, \mathrm{CH}_{4}, \mathrm{CD}_{4}\right)$ and atomic $(\mathrm{Ar}, \mathrm{Kr})$ species [14] offers a unique opportunity for such studies. One of the best studied orientational glass sys- tems is the $\mathrm{N}_{2}-\mathrm{Ar}$ mixture [37]. Pure $\mathrm{N}_{2}$ has two low-pressure crystalline forms, the hexagonal closepacked $(h c p)$ high temperature phase and the cubic $P a 3$ ( $f c c$ ) low temperature phase. Despite intrinsic geometrical frustration, pure $\mathrm{N}_{2}$ undergoes a first-order phase transition to a long-range periodic orientationally ordered $\alpha$-phase below $T_{\alpha \beta}=35.6 \mathrm{~K}$; the high temperature $\beta$-phase is orintationally disordered.

Solid $\left(\mathrm{N}_{2}\right)_{1-x} \mathrm{Ar}_{x}$ is obtained by simply cooling liquid mixtures, as nitrogen and argon are completely miscible. As the Ar concentration $x$ is increased, the $h c p$-to-fcc transition temperature decreases. Above the critical Ar concentration $x_{c} \approx 0.23$, the $h c p$ lattice appears to be stable down to $T=0$. The dynamical orientational disorder of the high- $T$ phase eventually freezes into a static pattern of randomly oriented $\mathrm{N}_{2}$ molecules, the orientational glass [37].

Being a mixture of insulators, the $\mathrm{N}_{2}-\mathrm{Ar}$ system has a very large energy gap $(\sim 10 \mathrm{eV})$, so that even at high temperature the ambient density of free electronic states is exponentially low. Experimental study of electron transport in this system therefore requires that the empty conduction band be «injected» with free carriers, ideally in low enough concentrations that electron-electron interactions can be safely ignored. The ionization of molecules and/or atoms by high energy charged particles (e.g. positive muons) offers just such a source of free carriers.

Figure 5 depicts the temperature dependences of the asymmetries (amplitudes) of the various signals in solid $\left(\mathrm{N}_{2}\right)_{1-x} \mathrm{Ar}_{x}$ for $x=0,0.09,0.16$, and 0.25 . At high temperature (above about $40 \mathrm{~K}$ ), all the mixtures have roughly the same $\mathrm{Mu}$ and $\mu_{D}$ asymmetries as pure $\mathrm{N}_{2}$. At low temperatures, however, adding argon causes dramatic changes. In pure $\mathrm{N}_{2}$ below about $30 \mathrm{~K}$ there is a large $\mathrm{Mu}$ signal and a small $\mu_{D}$ signal, indicating efficient DMF; as $\mathrm{Ar}$ is added there is a progressively larger $\mu_{D}$ signal, indicating reduced DMF, until at $x=0.25$ there is only a small Mu signal.

In solid $\mathrm{N}_{2}$ muonium formation has been shown $[38,39]$ to proceed via two channels: the thermal DMF process outlined above and the epithermal prompt process which takes place prior to the $\mu^{+}$ thermalization and is therefore independent of temperature, electron mobility etc. The small, temperature independent $\mathrm{Mu}$ amplitude in the $x=0.25$ sample (see Fig. 5) is the same as the prompt $\mathrm{Mu}$ amplitude in pure solid nitrogen [39], suggesting a complete absence of DMF in the orientational glass.

The hypothesis that $\mathrm{Mu}$ formation in the $x=0.25$ mixture is essentially all via the prompt channel at $20 \mathrm{~K}$ is further supported by the observation that $A_{\mathrm{Mu}}$ and $A_{D}$ do not depend on an externally applied electric field for that sample, as shown in Fig. 6. Both amplitudes show 


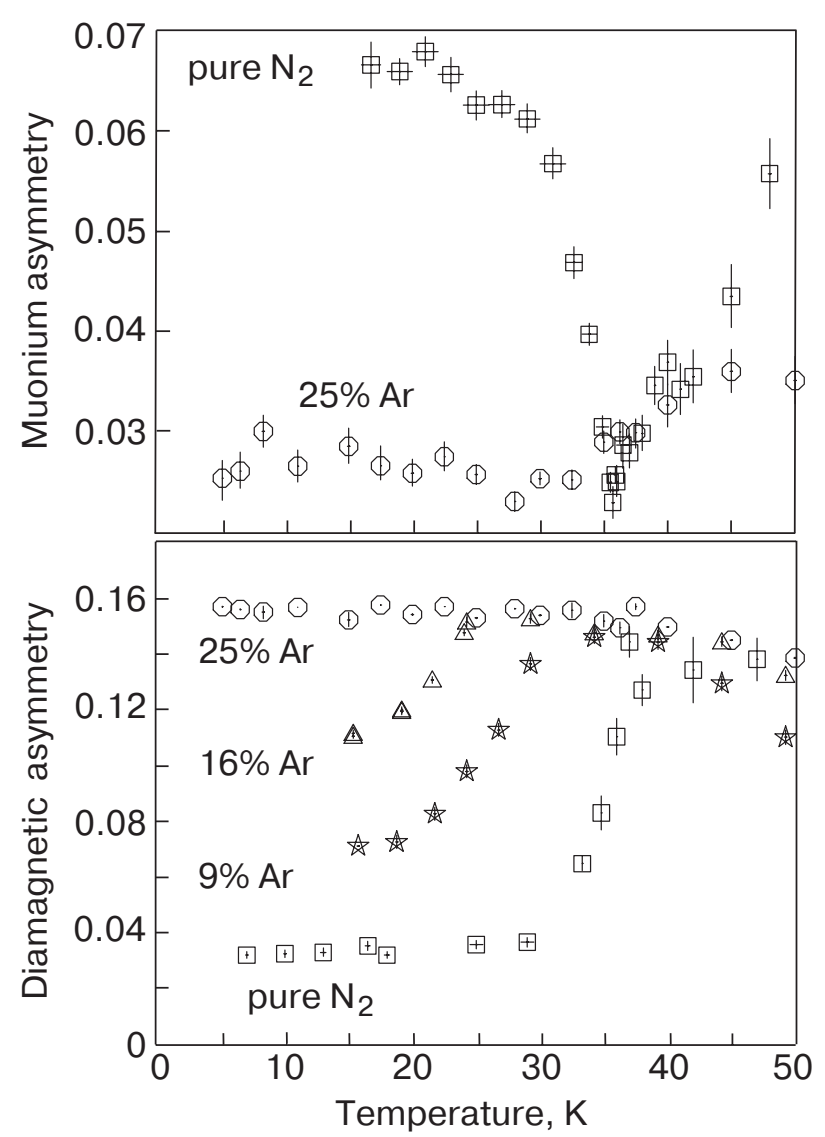

Fig. 5. Temperature dependences of muonium (top, $\mathrm{H} \approx$ $\approx 5 \mathrm{G}$ ) and net diamagnetic (bottom, $\mathrm{H} \approx 100 \mathrm{G}$ ) signal amplitudes in pure solid nitrogen (squares) and solid $\left(\mathrm{N}^{2}\right)_{1-x} \operatorname{Ar}_{x}$ (circles: $x=0.25$; triangles: $x=0.16$; stars: $x=0.09)$.

significant electric field dependence in pure $\mathrm{N}_{2}$ at 20 $\mathrm{K}$, from which the characteristic muon-electron distance is estimated to be about $5 \cdot 10^{-6} \mathrm{~cm}[38,39]$; about the same value of muon-electron distance is estimated in solid Ar, which exhibits almost $100 \%$ DMF [18]. The absence of DMF at this length scale at low temperature in the $x=0.25$ mixture suggests that electrons are localized in orientational glass [13].

\section{Conclusions}

Scaling from light to heavy CRG enabled us to reveal new features of end-of-track motion in these substances.

It is shown that unique properties of liquid helium - big relative mobility and light relative mass of positive ions make it outstanding in comparison with other condensed gases.

In solid $\mathrm{Ne}$ and $\mathrm{Ar}$ (where electrons are delocalized and light) track seems to be linear chain of anions ended by muon and surrounded by the cloud of electrons. The number of electrons involved in muonium formation is about ten in solid $\mathrm{Ne}$ and probably bigger in

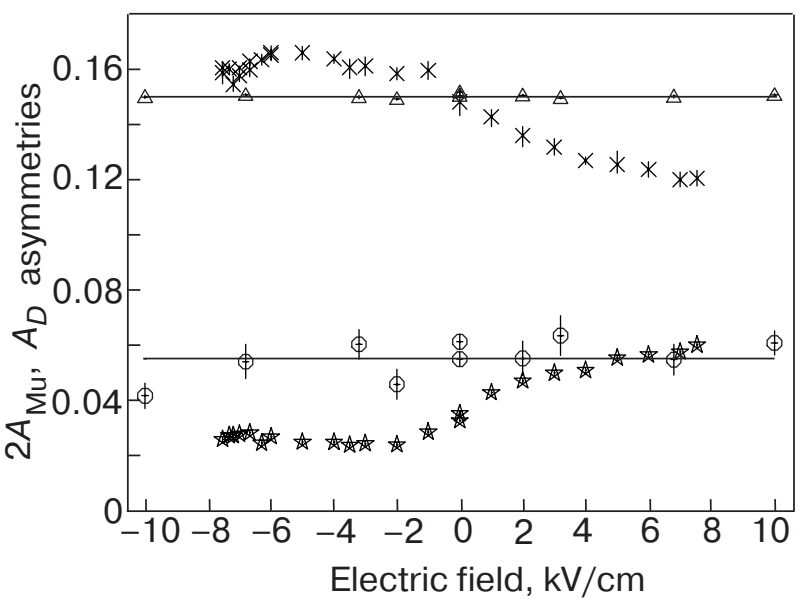

Fig. 6. Electric field dependences of $2 A_{\mathrm{Mu}}$ and $A_{D}$ in pure solid nitrogen (crosses and stars, respectively) and in solid $75 \% \mathrm{~N}_{2}+25 \% \mathrm{Ar}$ (circles and triangles, respectively) in a transverse magnetic field $H=36 \mathrm{G}$ at $T=20 \mathrm{~K}$. The muonium amplitudes are doubled to compensate for the $50 \%$ depolarization of $\mathrm{Mu}$ by hyperfine oscillations [16].

solid Ar. From comparison of Ne and Ar data we can estimate the distance between track anions about $10^{-6} \mathrm{~cm}$ and the square average distance between electron and parent anion about $10^{-5} \mathrm{~cm}$.

A slight difference between muonium formation in liquid and solid Ar (electrons are delocalized in both phases) could be attributed to the presence of crystalline defects in the solid.

In liquid Ne muon can recombine with delocalized or localized electrons. Localization of initially free track electrons in liquid results in high (in compression with solid $\mathrm{Ne}$ ) internal electric fields. External electric fields prevent electron localization.

We have demonstrated a dramatic effect of orientational disorder on electron transport in an insulator. In contrast to electron delocalization in the orientationally ordered phase of $\alpha-\mathrm{N}_{2}$, electrons appear to be localized in the orientational glass $\left(\mathrm{N}_{2}\right)_{0.75} \mathrm{Ar}_{0.25}$.

\section{Acknowledgments}

This work was supported by the Canadian Institute for Advanced Research, the Natural Sciences and Engineering Research Council of Canada, the National Research Council of Canada, and the Engineering and Physical Sciences Research Council of the United Kingdom. Two of us (VGS and DGE) were also supported by the INTAS Foundation (through grant 97-30063) and the Royal Society (through the International Award), NATO (through grant PST.CLG.977687). We would like to thank Prof. V.N. Gorelkin for useful discussions. DGE and VGS also express their gratitude to Profs. 
S.T. Belyaev, V.P. Martemyanov, and V.A. Matveev for constant support.

1. R.A. Muller, S.E. Derenzo, G. Smadja, D.B. Smith, R.G. Smits, H. Zaklad, and L.W. Alvarez, Phys. Rev. Lett. 27, 532 (1971).

2. E. Morenzoni, F. Kottmann, D. Maden, B. Matthias, M. Meyberg, Th. Prokscha, Th. Wutzke, and U. Zimmermann, Phys. Rev. Lett. 72, 2793 (1994).

3. W.E. Spear and P.G. Le Comber, in: Rare Gas Solids, M.L. Klein and J.A. Venables (eds.), Academic Press, New York (1977), p. 1119.

4. L.S. Miller, S. Howe, and W.E. Spear, Phys. Rev. 166, 871 (1968).

5. B.E. Springett, J. Jortner, and M.H. Cohen, J. Chem. Phys. 48, 2720 (1968).

6. A.T. Golov and L.P. Mezhov-Deglin, JETP Lett. 56, 514 (1992).

7. L. Bruschi, G. Mazzi, and M. Santini, Phys. Rev. Lett. 28, 1504 (1972).

8. R.J. Loveland, P.G. Le Comber, and W.E. Spear, Phys. Rev. B6, 3121 (1972).

9. Y. Sakai, W.F. Schmidt, and A. Khrapak, Chem. Phys. 164, 139 (1992).

10. A.O. Caldeira and A.J. Leggett, Phys. Rev. Lett. 46, 211 (1981); Ann. Phys. (N.Y.) 149, 374 (1983).

11. P.W. Anderson, Phys. Rev. 109, 1492 (1958).

12. N.F. Mott, Metal-Insulator Transitions, Taylor and Francis, London (1974)

13. V. Storchak, D.G. Eshchenko, J.H. Brewer, G.D. Morris, S.P. Cottrell, and S.F.J. Cox, Phys. Rev. Lett. 85, 166 (2000).

14. U.T. Hochli, K. Knorr, and A. Loidl, Adv. Phys. 39, 405 (1990).

15. H. Schnyders, S.A. Rice, and L. Meyer, Phys. Rev. Lett. 15, 187 (1965).

16. A Schenck, Muon Spin Rotation: Principles and Applications in Solid State Physics, Adam Hilger, Bristol (1986); S.F.J. Cox, J. Phys. C20, 3187 (1987); J.H. Brewer, in: Encyclopedia of Applied Physics 11, VCH, New York (1995), p. 23.

17. M. Senba, J. Phys. B21, 3093 (1988); ibid. B22, 2027 (1989); ibid. B23, 1545 (1990).

18. V.G. Storchak, J.H. Brewer, and D.G. Eshchenko, Appl. Mag. Reson. 13, 15 (1997).

19. D.G. Eshchenko, Ph.D. Thesis, Kurchatov Institute, Moscow, 1996 (in Russian).

20. V. Storchak, J.H. Brewer, and G.D. Morris, Phys. Rev. Lett. 75, 2384 (1995).
21. P.W. Percival, E. Roduner, and H. Fischer, Chem. Phys. 32, 353 (1978); D.C. Walker, Muon and Muonium Chemistry, Cambridge Univ. Press (1983); F.M. Jacobsen, Hyperfine Interactions 32, 501 (1986); O.E. Mogensen and P.W. Percival, Radiat. Phys. Chem. 28, 85 (1986).

22. E. Krasnoperov, E. Meilikhov, R. Abela, D. Herlach, E.Morenzoni, F. Gygax, A. Schenck, and D. Eshchenko, Phys. Rev. Lett. 69, 1560 (1992).

23. V. Storchak, J.H. Brewer, and G.D. Morris, Phys. Rev. Lett. 76, 2969 (1996).

24. M. Inokuti, Rev. Mod. Phys. 43, 297 (1971).

25. V.M. Gorelkin and L.P. Kotova, Hyperfine Interac. 65, 841 (1990).

26. L.D.A. Siebbeles, S.M. Pimblott, and S.F.J. Cox, J. Chem. Phys. 111, 7493 (1999); ibid., Physica B289-290, 404 (2000).

27. E.P. Krasnoperov, E.E. Meilikhov, D.G. Eshchenko, D. Herlach, E. Morenzoni, U. Zimmermann, F.N. Gygax, and A. Schenck, Hyperfine Interac. 87, 1011 (1994).

28. D.G. Eschenko, V.G. Storchak, and G. Morris, Phys. Lett. A264, 226 (1999).

29. E.P. Krasnoperov, E.E. Meilikhov, C. Baines, D. Herlach, G. Solt, U. Zimmermann, and D.G. Eshchenko, Hyperfine Interac. $97 / 98,347$ (1996).

30. A.J. Dahm and T.M. Sanders, J. Low. Temp. Phys. 2, 199 (1970).

31. F. Reif, L. Meyer, Phys. Rev. 119, 1164 (1960).

32. K.P. Atkins, Phys. Rev. 116, 1339 (1959).

33. T. Ellis, P.V.E. McClintock, R.M. Bowley, and D.R. Allum, Philos. Trans. R. Soc. London 296, A1425, 581 (1980).

34. A.J. Dahm, Charge Motion in Solid Helium, in: Progress in Low Temperature Physics, Vol. IX, North-Holland, Amsterdam (1985), p. 9.

35. E.P. Krasnoperov, E.E. Meilikhov, A.N. Ponamarev, V.Yu. Pommyakushin, D.G. Eshchenko, V.N. Duginov, V.A. Zhukov, T.N. Mamedov, and V.G. Olshevskij, JETP Lett. 59, 749 (1994).

36. N.F. Mott and E.A. Davis, Electron Processes in Non-Crystalline Materials, Clarendon Press, Oxford (1979).

37. J.A. Hamida, E.B. Genio, and N.S. Sullivan, J. Low Temp. Phys. 103, 49 (1996).

38. V. Storchak, J.H. Brewer, and G.D. Morris, Phys. Rev. Lett. 75, 2384 (1995).

39. V. Storchak, J.H. Brewer, G.D. Morris, D.J. Arseneau, and M. Senba, Phys. Rev. B59, 10559 (1999). 